\title{
Determination of the Size of Irregular Particles Using Interferometric Out-of-Focus Imaging
}

\author{
Marc Brunel, Huanhuan Shen, Sébastien Coëtmellec, Gérard Gréhan, and Tony Delobel \\ UMR CNRS 6614 CORIA, Département d'Optique et Laser, 675 Avenue de l'Université, 76801 Saint-Etienne du Rouvray Cedex, France \\ Correspondence should be addressed to Marc Brunel; marc.brunel@coria.fr
}

Received 30 July 2013; Revised 3 December 2013; Accepted 4 December 2013; Published 9 January 2014

Academic Editor: Adrian Podoleanu

Copyright (C) 2014 Marc Brunel et al. This is an open access article distributed under the Creative Commons Attribution License, which permits unrestricted use, distribution, and reproduction in any medium, provided the original work is properly cited.

\begin{abstract}
We present a mathematical formalism to predict speckle-like interferometric out-of-focus patterns created by irregular scattering objects. We describe the objects by an ensemble of Dirac emitters. We show that it is not necessary to describe rigorously the scattering properties of an elliptical irregular object to predict some physical properties of the interferometric out-of-focus pattern. The fit of the central peak of the $2 \mathrm{D}$ autocorrelation of the pattern allows the prediction of the size of the scattering element. The method can be applied to particles in a size range from a tenth of micrometers to the millimeter.
\end{abstract}

\section{Introduction}

Interferometric laser imaging for droplet or bubble sizing is a very convenient technique. It is relatively simple to develop and gives accurate evaluations of the size of these scattering particles in a wide range of configurations [17]. In addition, real-time algorithms can be developed to investigate a whole image directly using Fourier transforms instead of investigating the interferogram droplet per droplet or bubble per bubble [8]. Unfortunately, this technique is limited to the analysis of simple scattering objects as spherical droplets or bubbles. The deformation of these objects can affect significantly the interferometric patterns (orientation and frequency of the fringes) and the analysis of complex irregular objects does not seem to be possible. In this paper, we show however that interferometric out-of-focus imaging can allow the determination of some simple morphological information of irregular particles (size and global form of the envelope).

It is well established that interferometric out-of-focus imaging of droplets is based on the observation of interferences produced by the two "glare points" produced by the illumination beam on the droplet $[9,10]$. Under a geometrical optics approximation, these emitters correspond to the ray reflected on the particle and the ray refracted within the droplet [11]. These emitters can be further defined using
Lorenz-Mie theory expressed in terms of Debye series [10]. In the case of droplets in air, the scattering angle of $66^{\circ}$ is currently chosen. It is indeed the angle leading to the amplitudes of the reflected beam and the refracted beam having the same magnitude. The interferences that are recorded present then a contrast reaching unity.

For irregular particles, there is unfortunately no general solution to the field scattered by the particles when they are illuminated by a coherent plane wave, Gaussian beam, or laser sheet. The interferometric out-of-focus images are then more or less a speckle-like pattern. It is not possible to represent the scattering objects by a precise number of well-defined glare points as can be done with droplets or bubbles. To illustrate this, Figure 1 shows the interferometric out-of-focus image recorded on a CCD sensor. The experimental setup is as shown in Figure 2. A frequency-doubled Nd:YAG laser sheet illuminates $\mathrm{NaCl}$ salt crystals (the laser wavelength is $532 \mathrm{~nm}$ ). When observed with a microscope, these crystals have a global ellipsoidal form. The field scattered by these particles around the angle $\Theta=66^{\circ}$ is imaged with a lens (focus length $f=10 \mathrm{~cm}$ ). The distance between the crystals and the aperture is $z_{1}=8.5 \mathrm{~cm}$, the distance from the aperture to the lens is $z_{2}=6.5 \mathrm{~cm}$, and the distance from the lens to the CCD sensor is $z_{3}=20 \mathrm{~cm}$. The radius of the aperture is $R_{0}=4.8 \mathrm{~mm}$. We can observe in Figure $1 \mathrm{a}$ speckle-like pattern. Although it is not possible to describe 


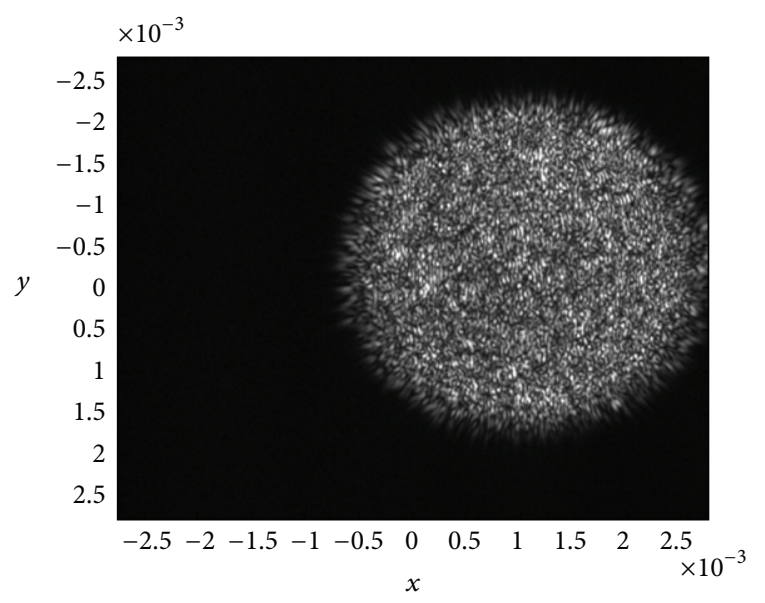

Figure 1: Experimental interferometric out-of-focus image of a $\mathrm{NaCl}$ salt crystal. All axes are in meters.

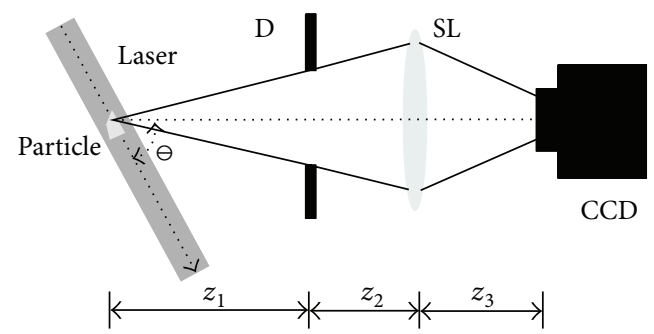

FIGURE 2: Experimental setup (schematic representation; the setup is not to scale).

rigorously the field scattered by the irregular scattering $\mathrm{NaCl}$ salt crystals, it does not mean that the speckle pattern does not contain rigorous information concerning the scattering particle.

In this paper, we will first develop in Section 2 a mathematical formalism to predict speckle-like interferometric outof-focus patterns created by irregular objects. The objects that we will describe are simplified to an ensemble of $N_{\mathrm{gp}}$ Dirac emitters located on a half sphere or more generally on a half ellipsoid. We will show that we can predict specklelike patterns that are observed experimentally. We will show that the size of the bright speck of the speckle patterns does not depend on the relative location of the glare points but depends on the dimension of the global half sphere or of the half ellipsoid. Finally, using this formalism, we will show in Section 3 that we can deduce the size of irregular $\mathrm{NaCl}$ salt crystal from the out-of-focus pattern of Figure 1.

\section{Simulation of Speckle-Like Interferometric Out-of-Focus Patterns}

From a theoretical point of view, the field emitted by a point Dirac emitter $i$, whose transverse position in the plane of the scattering particle is $\left(a_{i}, b_{i}\right)$, can be evaluated in the plane of the CCD sensor by generalized Huygens Fresnel integrals [12-15]. Their coefficients depend on the coefficients of optical transfer matrices describing the optical elements encountered between the particle and the sensor. The procedure has been detailed in references $[7,16]$ in the case of droplets represented by a pair of Dirac emitters and has been validated in all cases that could be tested experimentally $[7,17,18]$. For a unique Dirac emitter, we obtain the following expression of the electric field in the plane of the CCD sensor [7]:

$$
\begin{aligned}
& G_{i}(x, y, z) \\
& =\left(\left(\alpha_{i} e^{i \varphi_{i}} e^{i(2 \pi / \lambda)\left(n_{1} z_{1, i}+n_{2}\left(z_{2}+z_{3}\right)\right)} e^{i(\pi / \lambda)\left(\left(D_{2}^{x} x^{2} / B_{2}^{x}\right)+\left(D_{2}^{y} y^{2} / B_{2}^{y}\right)\right)}\right)\right. \\
& \left.\quad \times\left((i \lambda)^{2} \sqrt{B_{1, i}^{x} B_{1, i}^{y} B_{2}^{x} B_{2}^{y}}\right)^{-1}\right) \\
& \quad \times \sum_{k=1}^{N} \frac{P_{k} \pi e^{\beta_{i}(k)}}{\sqrt{\gamma_{x, i}(k) \gamma_{y, i}(k)}},
\end{aligned}
$$

where $z$ is the optical longitudinal axis of the imaging setup, $x$ and $y$ are the transverse coordinates in the plane of the CCD sensor, and

$$
\begin{gathered}
\beta_{i}(k)=i \frac{\pi A_{1, i}^{x} a_{i}^{2}}{\lambda B_{1, i}^{x}}+i \frac{\pi A_{1, i}^{y} b_{i}^{2}}{\lambda B_{1, i}^{y}}-\frac{\phi_{x, i}^{2}}{4 \gamma_{x, i}(k)}-\frac{\phi_{y, i}^{2}}{4 \gamma_{y, i}(k)}, \\
\gamma_{x, i}(k)=\frac{Q_{k}}{R_{0}^{2}}-i \frac{\pi}{\lambda}\left(\frac{D_{1, i}^{x}}{B_{1, i}^{x}}+\frac{A_{2}^{x}}{B_{2}^{x}}\right), \\
\gamma_{y, i}(k)=\frac{Q_{k}}{R_{0}^{2}}-i \frac{\pi}{\lambda}\left(\frac{D_{1, i}^{y}}{B_{1, i}^{y}}+\frac{A_{2}^{y}}{B_{2}^{y}}\right), \\
\phi_{x, i}(k)=\frac{2 \pi}{\lambda}\left(\frac{a_{i}}{B_{1, i}^{x}}+\frac{x}{B_{2}^{x}}\right) \\
\phi_{y, i}(k)=\frac{2 \pi}{\lambda}\left(\frac{b_{i}}{B_{1, i}^{y}}+\frac{y}{B_{2}^{y}}\right) .
\end{gathered}
$$

The different $A_{1, i}^{p}, B_{1, i}^{p}, C_{1, i}^{p}$ and $D_{1, i}^{p}$ (with $p=x$ or $p=y$ ) are the coefficients of the optical transfer matrices between the emitter $i$ and the plane where the aperture is located. $n_{1} z_{1, i}$ is the optical path between the emitter $i$ and the plane of the aperture. We can define two different transfer matrices for both transverse axes $x$ and $y$, which allows us to describe cylindrical geometries. The transmission coefficient of the aperture equals 1 within a disk of radius $R_{0}$ and equals 0 outside. It is expressed as a superposition of 10 Gaussian functions, as was proposed by Wen and Breazeale [19]. The corresponding coefficients of this decomposition $P_{k}$ and $Q_{k}$ can be found in [19]. The $A_{2}^{p}, B_{2}^{p}, C_{2}^{p}$, and $D_{2}^{p}$ (with $p=x$ or $p=y$ ) are the coefficients of the optical transfer matrices between the plane of the aperture and the plane of the CCD sensor. $n_{2}\left(z_{2}+z_{3}\right)$ is the optical path between the plane of the aperture and the plane of the CCD sensor.

In ILIDS, it has been shown that a droplet can be well represented by two emitters $[7,16]$. Let us now consider 


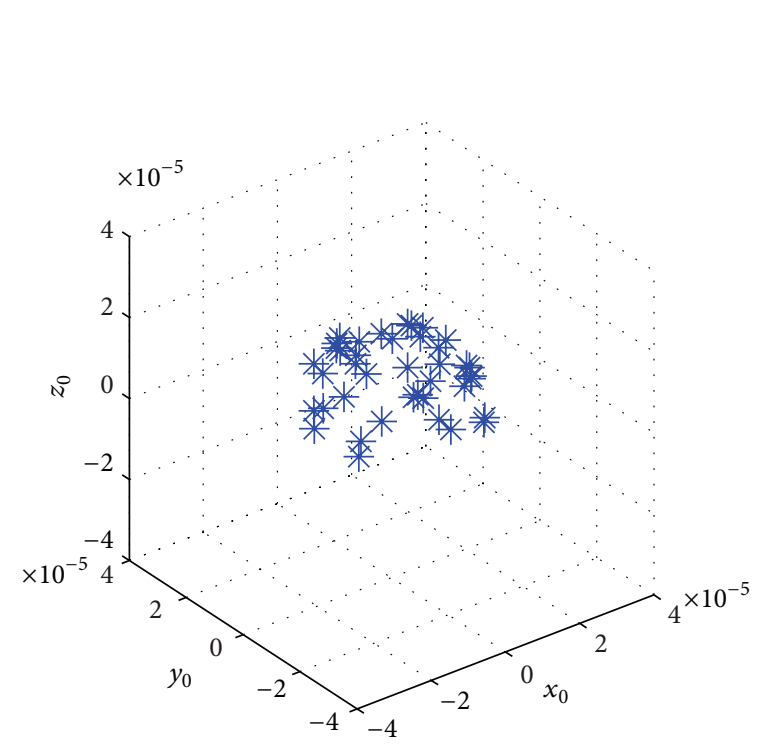

(a)

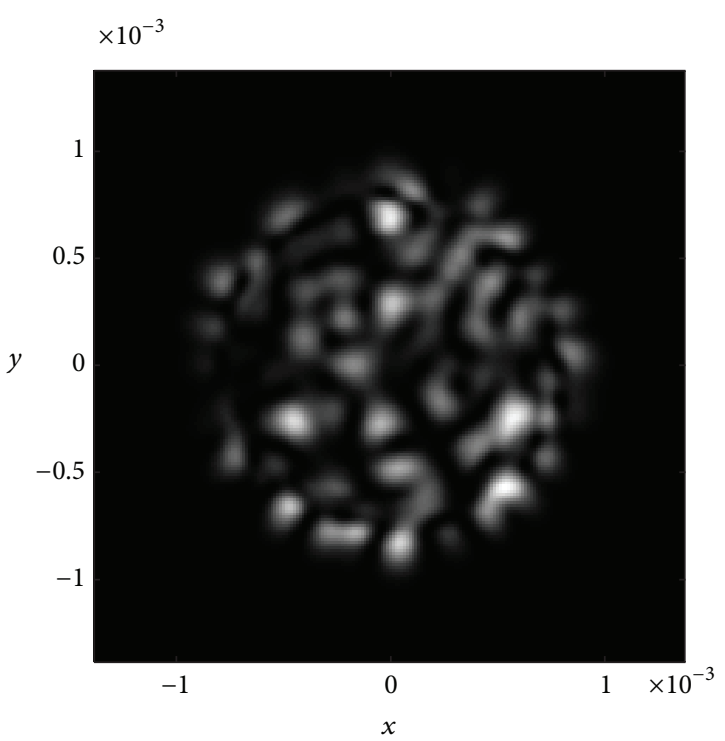

(b)

FIGURE 3: Half sphere composed of 40 randomly located Dirac emitters (a) and interferometric out-of-focus image created by this object (b). The radius of the half sphere is 20 micrometers. All axes are in meters.

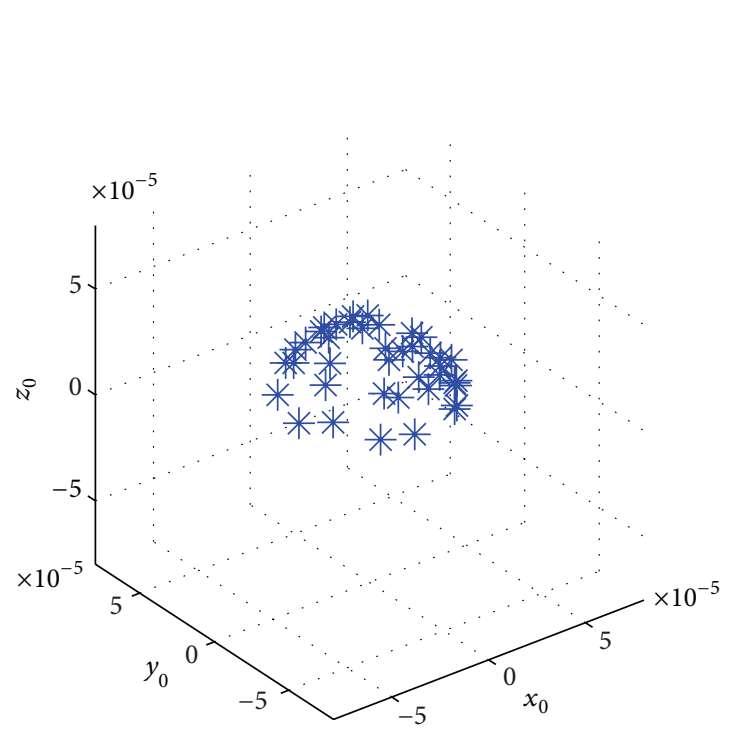

(a)

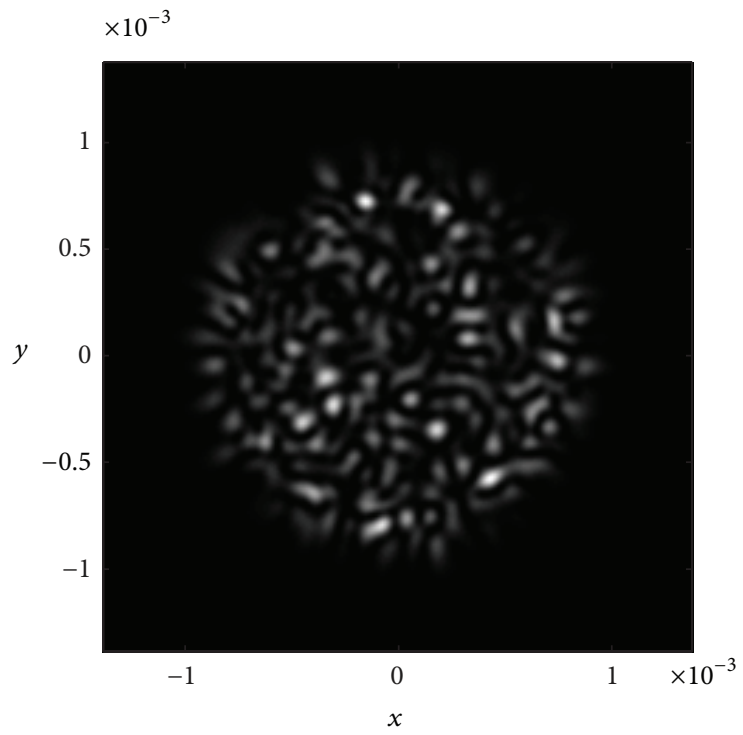

(b)

FIGURE 4: Half sphere composed of 40 randomly located Dirac emitters (a) and interferometric out-of-focus image created by this object (b). The radius of the half sphere is 40 micrometers. All axes are in meters.

the case of a multiemitter composed of $N_{\mathrm{gp}}$ Dirac emitters randomly located on a half sphere, as shown in Figure 3(a). The pattern observed on the CCD sensor can then be theoretically calculated. According to previous relations, the expression of the electric field in the CCD sensor plane can be written as follows:

$$
G_{\text {tot }}(x, y, z)=\sum_{i=1}^{N_{\mathrm{gp}}} G_{i}(x, y, z) .
$$

In the next simulations, we will assume that the amplitude of the electric field is the same for all emitters $\left(\alpha_{i}=1\right.$ in normalized units). In addition, we are interested in the characterization of particles in the size domain from a tenth of micrometers to hundreds of micrometers. For spherical droplets, the phase shift introduced between the two glare points (which represent the reflected beam and the beam refracted by the droplet without any internal reflection) is then much higher than $2 \pi$. In the case of irregular particles, 


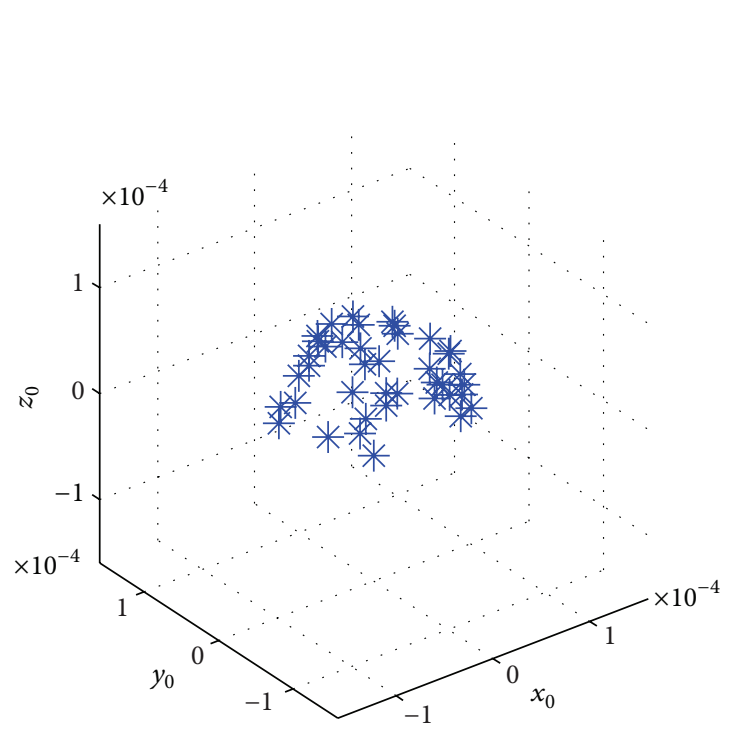

(a)

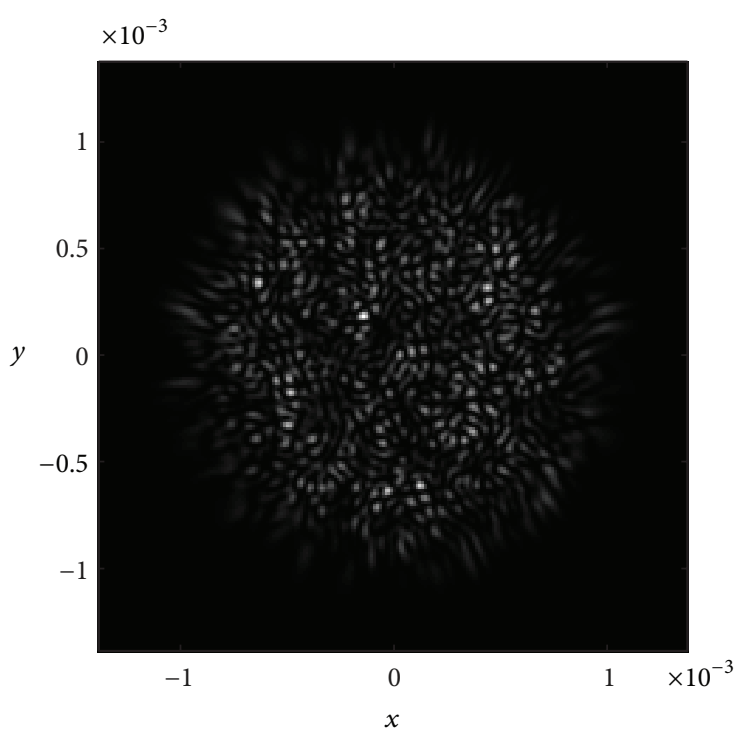

(b)

FiguRE 5: Half sphere composed of 40 randomly located Dirac emitters (a) and interferometric out-of-focus image created by this object (b). The radius of the half sphere is 80 micrometers. All axes are in meters.

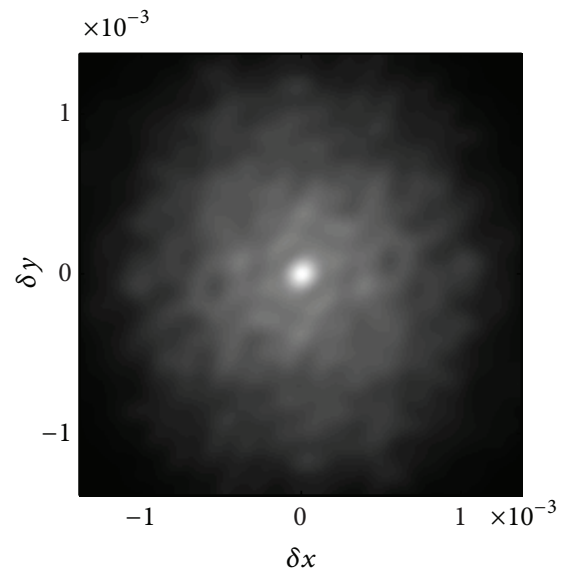

(a)

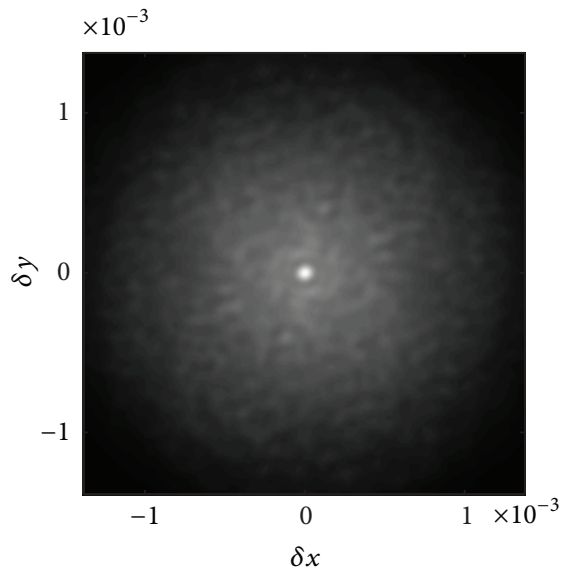

(b)

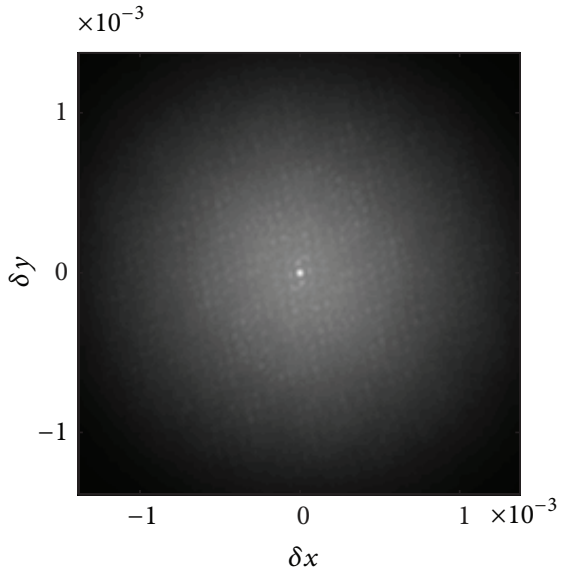

(c)

FiguRE 6: 2D autocorrelations of the interferometric out-of-focus patterns of Figures 3(b), 4(b), and 5(b), respectively. All axes are expressed in meters.

we will thus assume that the phase $\varphi_{i}$ of each emitter is a random variable in the range $[0,2 \pi]$.

We present in Figure 3 the geometrical repartition of $N_{\text {gp }}=40$ Dirac emitters randomly located on a half sphere of radius of 20 micrometers (Figure 3(a)) and the interferometric out-of-focus image created by this object (Figure 3(b)) using previous relations (1)-(3). The imaging system as shown in Figure 2 is a lens whose focus length and radius are $f=10 \mathrm{~cm}$ and $R_{0}=10 \mathrm{~mm}$, respectively. The distance between the center of the half sphere and the aperture is $z_{1}=13 \mathrm{~cm}$, the distance from the aperture to the lens is $z_{2}=0 \mathrm{~cm}$ (the lens is the aperture in this case), and the distance from the lens to the CCD sensor is $z_{3}=39.3 \mathrm{~cm}$. We obtain a speckle-like pattern as in the case of the experimental image of Figure 1, although the dimension of the speck is different. For comparison, Figure 4 shows the geometrical repartition of $N_{\mathrm{gp}}=40$ Dirac emitters randomly located on a half sphere of radius of 40 micrometers (Figure 4(a)) and the interferometric out-of-focus image created by this object (Figure 4(b)). Finally, Figure 5 shows the geometrical repartition of $N_{\mathrm{gp}}=40$ Dirac emitters randomly located on a half sphere of radius 80 micrometers (Figure 5(a)) and the interferometric out-of-focus image created by this object (Figure 5(b)). The imaging system is the same in all three cases of Figures 3, 4, and 5. To characterize the size of the speck of a speckle pattern, it is interesting to plot the 2D-autocorrelation of this speckle pattern. Figures 6(a), 6(b), and 6(c) show the 2D autocorrelations of the 


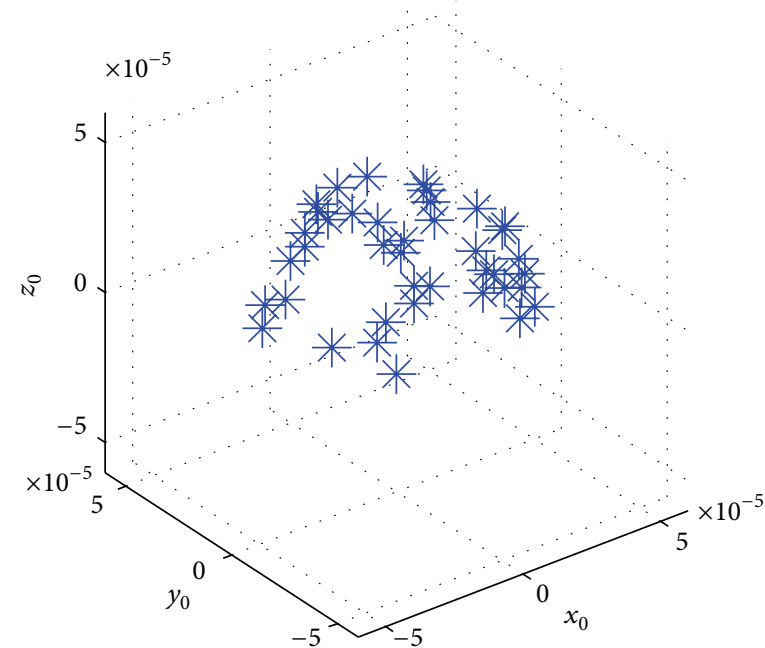

(a)

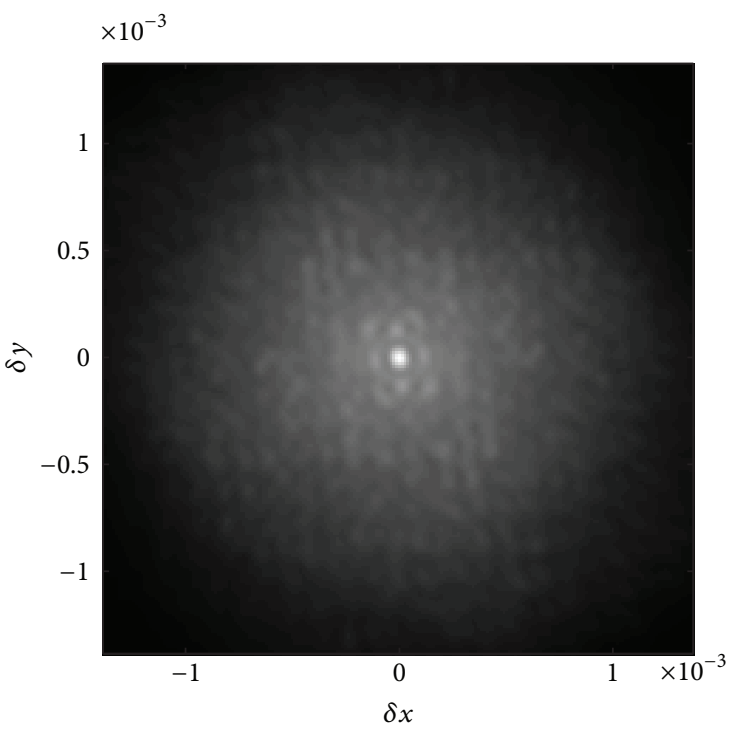

(c)

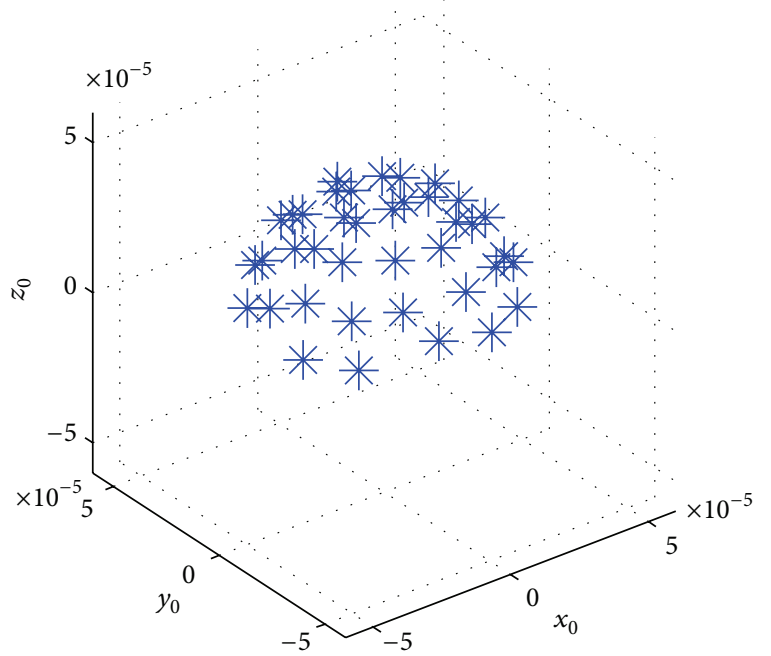

(b)

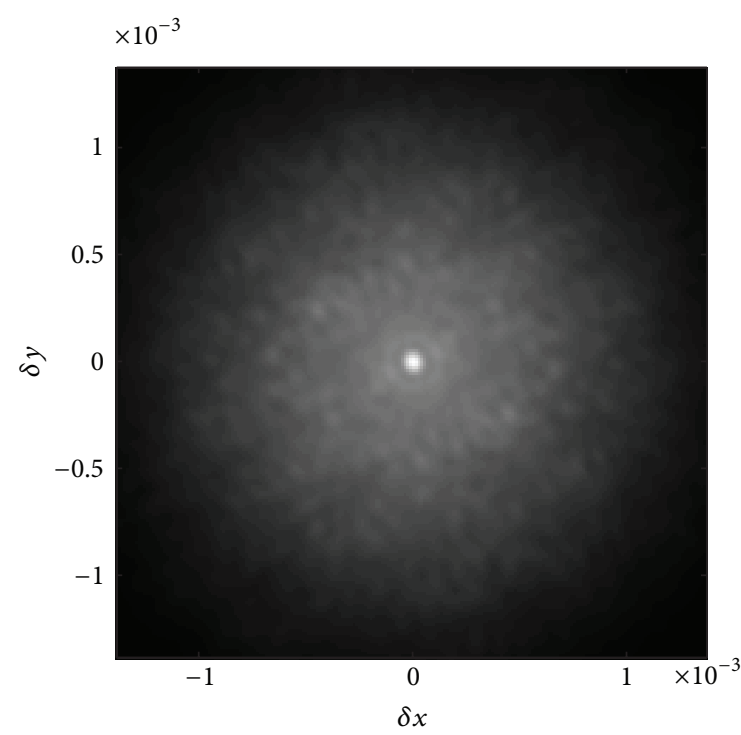

(d)

FIGURE 7: Half spheres composed of 40 Dirac emitters randomly located (a) or uniformly located over the half sphere (b). Autocorrelations of the speckle-like interferometric out-of-focus patterns that they create: (c) and (d), respectively. The radius of the half spheres is 40 micrometers. All axes are expressed in meters.

three speckle patterns presented in Figures 3(b), 4(b), and 5 (b), respectively. We observe a thin central peak which characterizes the size of the bright speck. We can clearly observe that the higher the dimension of the sphere, the smaller the size of the speck of the speckle pattern.

It is now important to know whether this characteristic of the speckle-like patterns, that is, the size of the bright speck, depends on the relative location of the emitters to each other. We present thus in Figures 7 and 8 a comparison of the results obtained when the Dirac emitters are located randomly or uniformly on a half sphere of radius of 40 micrometers. The setup parameters are those of previous Figures 3, 4, 5, and 6. First, Figures 7(a) and 7(b) present the location of the $N_{\mathrm{gp}}=40$ glare points on the half sphere when the emitters are randomly located (Figure 7(a)) or uniformly located (Figure 7(b)). We have then calculated the out-offocus patterns created by these objects using relations (1)(3). Their 2D autocorrelations are presented in Figures 7(c) and $7(\mathrm{~d})$, respectively. Although both $2 \mathrm{D}$ autocorrelations are not identical, we observe a central bright peak whose dimensions seem to be the same in both cases. To quantify this observation exactly, Figure 8(a) shows the $x$-cut of the central peak of the 2D-autocorrelation in the case of a random location of the emitters (black curve) and in the case of a uniform location of the emitters (red curve). In a similar way, Figure 8(b) shows the $y$-cut of the central peak of the $2 \mathrm{D}$-autocorrelation in the case of a random location of the emitters (black curve) and in the case of a uniform location of the emitters (red curve). Although the pedestals of the curves are not identical, the width of the central peak is the same for 


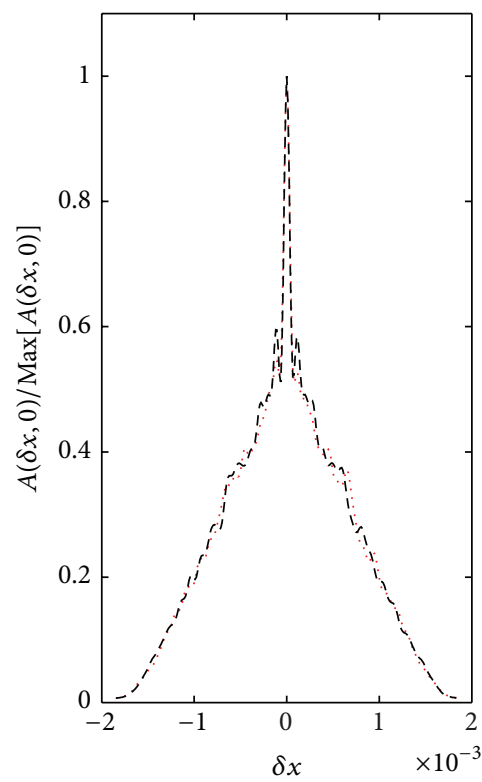

(a)

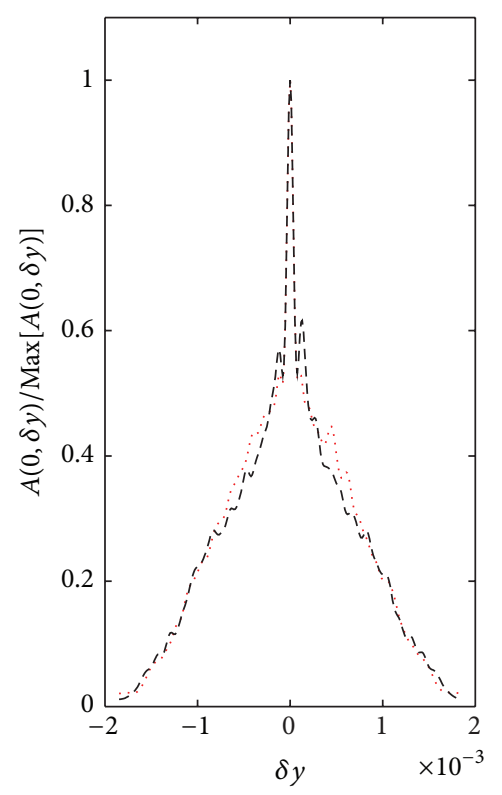

(b)

FIGURE 8: $x$-cut (a) and $y$-cut (b) of the central peak of the 2D-autocorrelation of the simulated speckle-like interferometric out-of-focus patterns for a random location of the emitters (black dashed lines) or a regular location of the emitters (red dotted lines). The $x$-axes are expressed in meters, while the $y$-axes represent normalized amplitudes (no unit).

a random or a uniform location of the emitters. This is true for both $x$-cuts and $y$-cuts of the peaks. In conclusion, the relative location of the emitters does not significantly affect the dimension of the central peak of the 2D-autocorrelation.

In a similar way, we could further illustrate that the number of emitters to be considered $(20,30,40, \ldots)$ does not affect significantly the dimension of the central peak of the $2 \mathrm{D}$-autocorrelation. In conclusion, it is not necessary to describe rigorously the scattering properties of an irregular object to predict some physical properties of the scattering pattern observed in an out-of-focus plane. In particular, the observation of the central peak of the $2 \mathrm{D}$-autocorrelation of the interferometric out-of-focus pattern allows us to predict the dimension of the scattering element by comparison with simulated patterns using our relations (1)-(3).

\section{Comparison with Experimental Data}

Finally, let us consider the experimental out-of-focus pattern presented in Figure 1. We have calculated the 2Dautocorrelation of this pattern (not reported here). As previously observed with our simulations, we observe a central peak on a large pedestal. We report in Figure 9 (a) the $x$-cut of the central peak of this 2D-autocorrelation (red curve), while Figure 9(b) shows the $y$-cut of the central peak of this $2 \mathrm{D}$-autocorrelation (red curve). We can see that the central peak of the 2D-autocorrelation is not circular. A zoom of this central peak shows that it is actually elliptical (with principal axes $x$ and $y$ ). It indicates that the scattering object (a $\mathrm{NaCl}$ salt crystal) cannot be viewed as a spherical object but as a rough ellipsoidal object as observed with a microscope. In order to simulate these results, we have used our relations (1)-(3) with the parameters that are necessary to simulate the experimental setup of Figure 2. Let us recall these parameters. The illumination wavelength is $\lambda=532 \mathrm{~nm}$. The imaging lens (SL in Figure 2) has a focus length $f=10 \mathrm{~cm}$. The aperture $D$ has a radius $R_{0}=4.8 \mathrm{~mm}$. The distance between the $\mathrm{NaCl}$ salt crystal and the aperture is $z_{1}=8.5 \mathrm{~cm}$, the distance from the aperture to the lens is $z_{2}=6.5 \mathrm{~cm}$, and the distance from the lens to the CCD sensor is $z_{3}=20 \mathrm{~cm}$. In the simulations, the matrices to be considered are matrices $M_{1, i}$ (coefficients $A_{1, i}, B_{1, i}, C_{1, i}$, and $D_{1, i}$ ) describing the first section of the imaging system, which takes into account propagation from the different emitters of the object to the aperture (see Figure 2), and a matrix $M_{2}$ (coefficients $A_{2}, B_{2}$, $C_{2}$, and $D_{2}$ ) which takes into account the propagation from the aperture to the lens, the transmission through the lens, and the propagation from the lens to the CCD sensor. The $\mathrm{NaCl}$ salt crystal is modeled by $N_{\mathrm{gp}}=60$ Dirac emitters uniformly located on a half ellipsoid. It is presented in Figure 10(a). The best fit of the experimental data is obtained for dimensions of the half ellipsoid: 800 micrometers along the $x$-axis and 380 micrometers along the $y$ - and $z$-axis. Note that the dimension along the $z$-axis (which is the optical axis of the experiment) has no significant influence on the planar interferometric out-of-focus pattern which is sensitive to the $x$-and $y$-dimensions. Figure 10(b) shows a zoom of the central peak of the 2D-autocorrelation of the interferometric pattern that is then calculated in the CCD sensor plane. The central peak is clearly elliptical, due to the ellipticity of the object around the $x$ - and $y$-axes. Finally, we report in black curves in Figure 9 the $x$-cut of the central peak of this 2Dautocorrelation (Figure 9(a)) and the $y$-cut of the central 


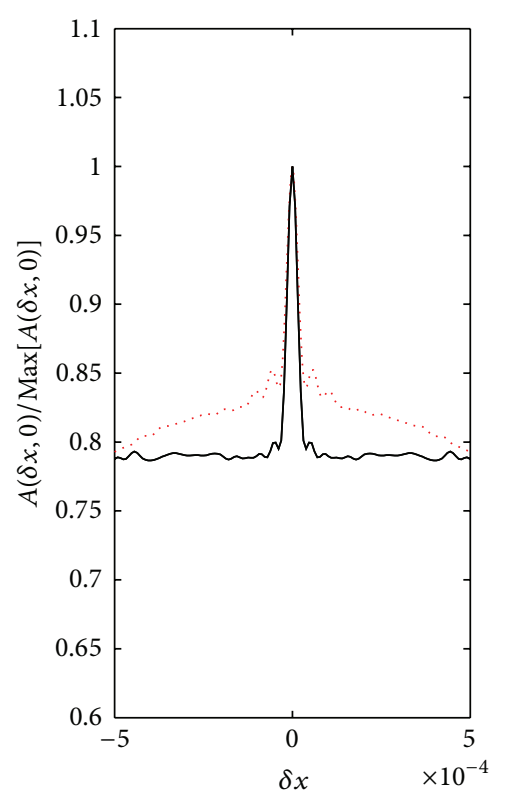

(a)

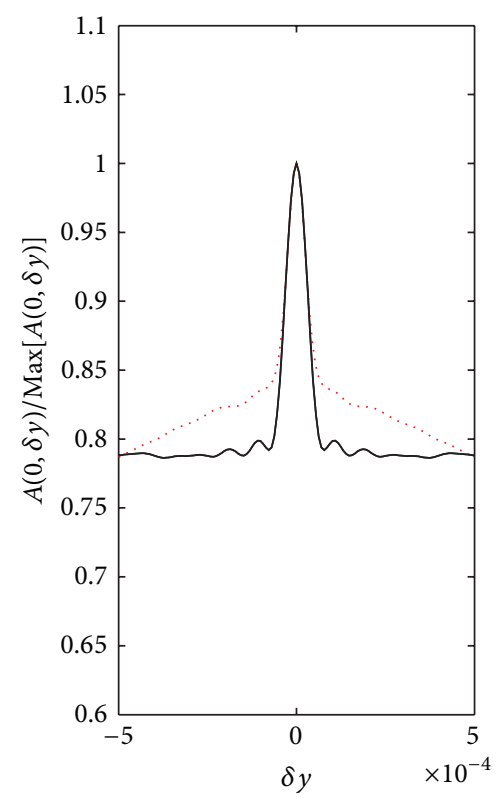

(b)

FIGURE 9: $x$-cuts (a) and $y$-cuts (b) of the central peak of the 2D autocorrelations of the experimental (dotted red lines) and simulated (solid black lines) speckle-like interferometric out-of-focus patterns. The $x$-axes are expressed in meters, while the $y$-axes represent normalized amplitudes (no unit).

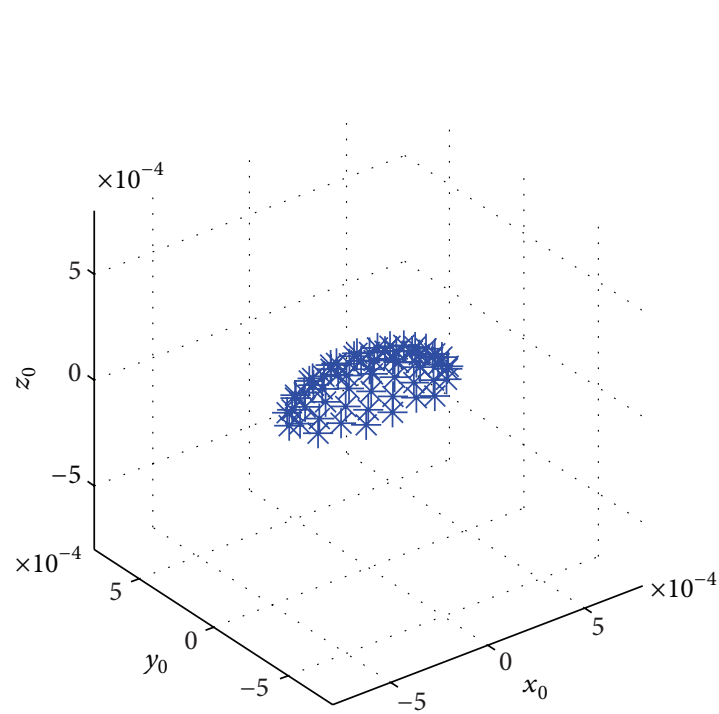

(a)

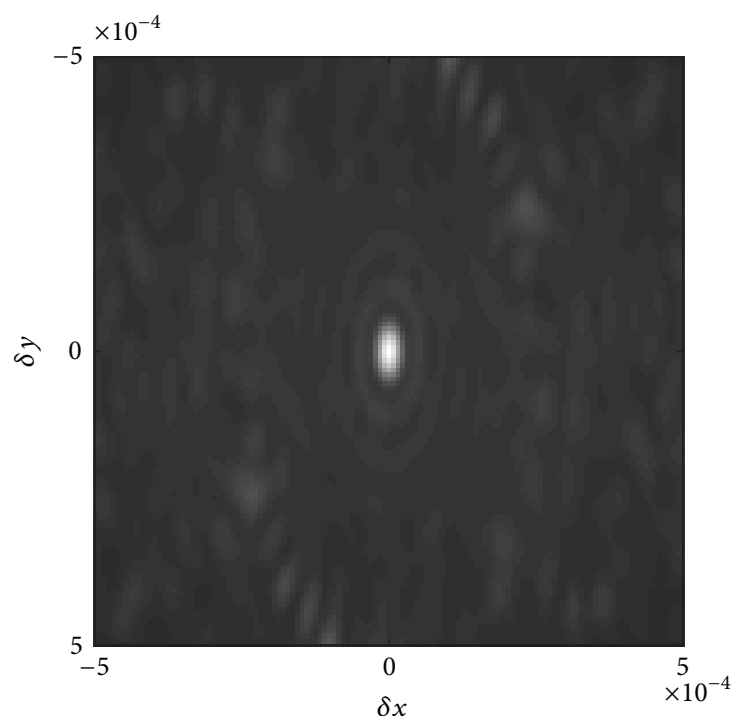

(b)

FIGURE 10: Half ellipsoid composed of 60 Dirac emitters uniformly located (a) and zoom of the central peak of the 2D-autocorrelation of the interferometric out-of-focus image created by this object (b). All axes are in meters.

peak of this 2D-autocorrelation (Figure 9(b)). We can see the very good concordance between experimental and simulated results concerning the dimensions of the central peak.

In conclusion, we confirm experimentally that the observation of the central peak of the $2 \mathrm{D}$-autocorrelation of the interferometric out-of-focus scattering pattern allows us to predict the dimensions of the scattering element by comparison with simulated patterns using our relations (1)-(3). This can be done despite not being able to describe rigorously the scattering properties of the rough irregular object. These first experiments show the potentiality of this technique with relatively simple irregular objects: ellipsoidal rough salt crystals. Future studies should show whether it is possible to characterize the morphology of more complex irregular objects.

It is very important to note that the defocus parameter needs to be sufficiently large, in such a way that the defocused 
images of all emitters overlap in the global out-of-focus image of the particle observed in the CCD sensor plane. This point is similar to classical ILIDS, where the defocus parameter needs to be sufficiently large, in such a way that the defocused images of both reflected and refracted glare points (in the case of a droplet) overlap to deliver interference fringes. This condition is respected in the results presented here.

\section{Conclusion}

In this paper, we have developed a mathematical formalism to predict speckle-like interferometric out-of-focus patterns created by ellipsoidal rough scattering objects. The objects that we describe are simplified to an ensemble of $N_{\mathrm{gp}}$ Dirac emitters located on global forms as half spheres or half ellipsoids. We could confirm experimentally that it is not necessary to describe rigorously the scattering properties of an elliptical irregular object to predict some physical properties of the scattering pattern observed in an out-offocus plane. In particular, the observation of the central peak of the 2D-autocorrelation of the interferometric out-of-focus pattern allows us to predict the dimensions of the scattering element by comparison with simulated patterns using our formalism. These first experiments show the possibility to characterize relatively simple irregular objects: ellipsoidal rough salt crystals. Future studies should show whether it is possible to characterize the morphology of more complex irregular objects. The formalism developed allows us to describe any imaging system as it is expressed versus the coefficients of the optical transfer matrices that describe the system. This method could have a wide number of applications, for example, the characterization of ashes in the atmosphere, or sediments in water.

\section{Conflict of Interests}

The authors declare that there is no conflict of interests regarding the publication of this paper.

\section{Acknowledgments}

The authors acknowledge the financial support of the French Agence Nationale de la Recherche (ANR), through the program Investissements d'Avenir (ANR-10-LABX-09-01) LabEx EMC3, and the European Commission through the EUFAR project (European Facility for Airborne Research).

\section{References}

[1] G. König, K. Anders, and A. Frohn, "A new light-scattering technique to measure the diameter of periodically generated moving droplets," Journal of Aerosol Science, vol. 17, no. 2, pp. 157-167, 1986.

[2] R. Ragucci, A. Cavaliere, and P. Massoli, "Drop sizing by laser light scattering exploiting intensity angular oscillation in the Mie regime," Particle \& Particle Systems Characterization, vol. 7, no. 4, pp. 221-225, 1990.

[3] K. H. Hesselbacher, K. Anders, and A. Frohn, "Experimental investigation of Gaussian beam effects on the accuracy of a droplet sizing method," Applied Optics, vol. 30, pp. 4930-4935, 1991.

[4] A. R. Glover, S. M. Skippon, and R. D. Boyle, "Interferometric laser imaging for droplet sizing: a method for droplet-size measurement in sparse spray systems," Applied Optics, vol. 34, no. 36, pp. 8409-8421, 1995.

[5] T. Kawaguchi, Y. Akasaka, and M. Maeda, "Size measurements of droplets and bubbles by advanced interferometric laser imaging technique," Measurement Science and Technology, vol. 13, no. 3, pp. 308-316, 2002.

[6] M. Maeda, Y. Akasaka, and T. Kawaguchi, "Improvements of the interferometric technique for simultaneous measurement of droplet size and velocity vector field and its application to a transient spray," Experiments in Fluids, vol. 33, no. 1, pp. 125-134, 2002.

[7] H. Shen, S. Coetmellec, G. Grehan, and M. Brunel, "ILIDS revisited: elaboration of transfer matrix models for the description of complete systems," Applied Optics, vol. 51, no. 22, pp. 5357-5368, 2012.

[8] A. Querel, P. Lemaitre, M. Brunel, E. Porcheron, and G. Gréhan, "Real-time global interferometric laser imaging for the droplet sizing (ILIDS) algorithm for airborne research," Measurement Science and Technology, vol. 21, no. 1, Article ID 015306, 2010.

[9] H. C. Van de Hulst and R. T. Wang, "Glare points," Applied Optics, vol. 30, no. 33, pp. 4755-4763, 1991.

[10] H. M. Nussenzveig, "High-frequency scattering by a transparent sphere. I. direct reflection and transmission," Journal of Mathematical Physics, vol. 10, pp. 82-124, 1969.

[11] W. J. Glantschnig and S. H. Chen, "Light scattering from water droplets in the geometrical optics approximation," Applied Optics, vol. 20, no. 14, pp. 2499-2509, 1981.

[12] C. Palma and V. Bagini, "Extension of the Fresnel transform to ABCD systems," Journal of the Optical Society of America A, vol. 14, no. 8, pp. 1774-1779, 1997.

[13] A. J. Lambert and D. Fraser, "Linear systems approach to simulation of optical diffraction," Applied Optics, vol. 37, no. 34, pp. 7933-7939, 1998.

[14] H. T. Yura and S. G. Hanson, "Optical beam wave propagation through complex optical systems," Journal of the Optical Society of America A, vol. 4, no. 10, pp. 1931-1948, 1987.

[15] M. Brunel, H. Shen, S. Coëtmellec, D. Lebrun, and K. Ait Ameur, "Phase contrast metrology using digital in-line holography: general models and reconstruction of phase discontinuities," Journal of Quantitative Spectroscopy and Radiative Transfer, vol. 126, pp. 113-121, 2013.

[16] M. Brunel and H. Shen, "Design of ILIDS configurations for droplet characterization," Particuology, vol. 11, no. 2, pp. 148-157, 2013.

[17] H. Shen, S. Coetmellec, and M. Brunel, "Cylindrical interferometric out-of-focus imaging for the analysis of droplets in a volume," Optics Letters, vol. 37, no. 19, pp. 3945-3947, 2012.

[18] H. Shen, S. Coëtmellec, and M. Brunel, "Simultaneous 3D location and size measurement of spherical bubbles using cylindrical interferometric out-of-focus imaging," Journal of Quantitative Spectroscopy and Radiative Transfer, vol. 131, pp. 153-159, 2013.

[19] J. J. Wen and M. Breazeale, "A diffraction beam expressed as the superposition of Gaussian beams," The Journal of the Acoustical Society of America, vol. 83, pp. 1752-1756, 1988. 

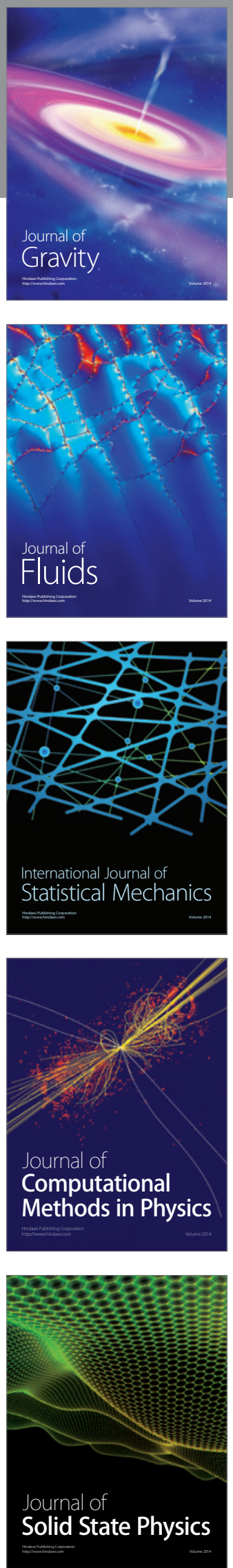

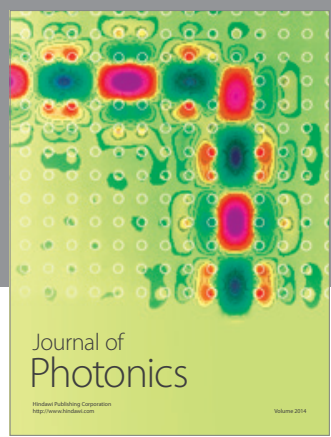

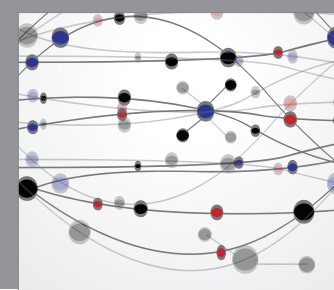

The Scientific World Journal

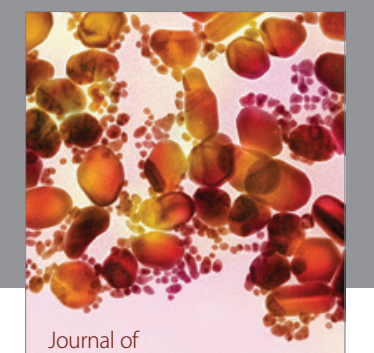

Soft Matter
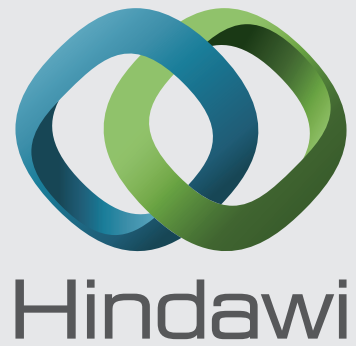

Submit your manuscripts at

http://www.hindawi.com
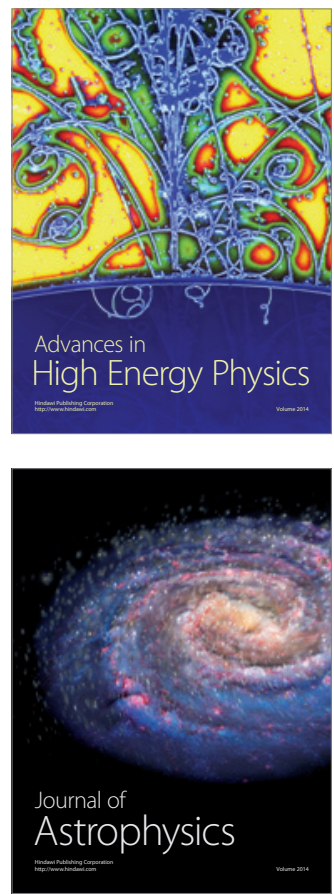
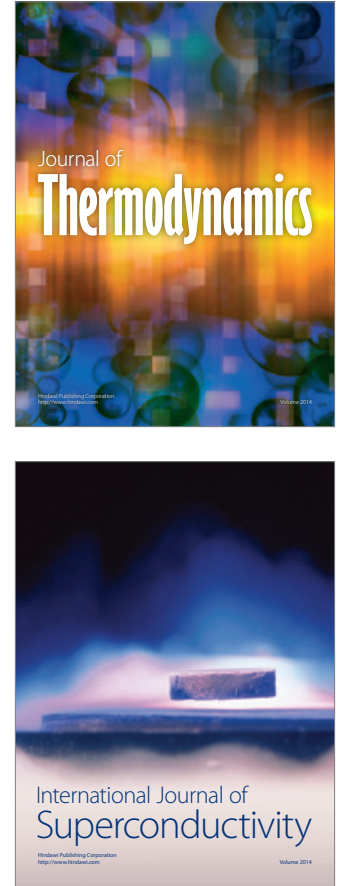
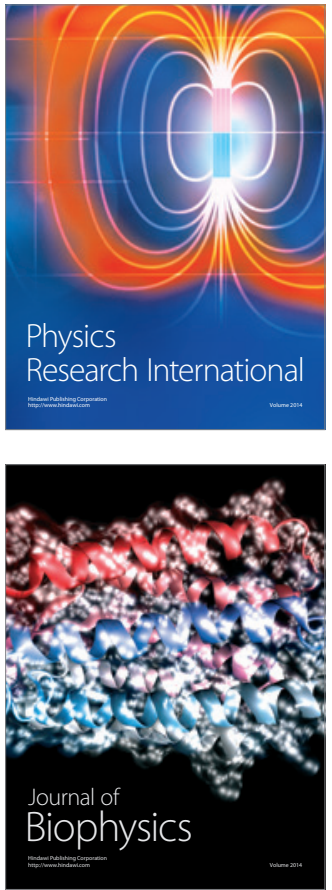
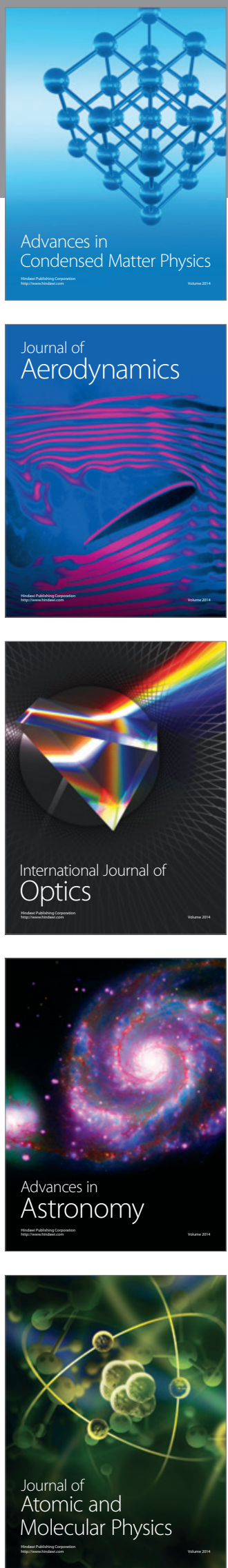